\title{
Enantioselective total syntheses of the novel tricyclic sesquiterpene hydrocarbons (+)- and (-)-kelsoene. Absolute configuration of the natural product
}

\author{
Goverdhan Mehta* and K. Srinivas \\ Department of Organic Chemistry, Indian Institute of Science, Bangalore 560 012, India
}

\begin{abstract}
Simple and preparatively efficacious lipase-catalysed kinetic resolution of endo,endo-cis-bicyclo[3.3.0]octane-2,6-diol rac $\mathbf{- 3}$ has provided ready access to bicyclo[3.3.0]octane-2,6-diones (-)-2 and (+)-2 of high enantiomeric purity. These $C_{2}$-symmetric diones have been further elaborated to the sesquiterpene hydrocarbon (+)-kelsoene 1 and ent-kelsoene (-)-1, respectively, thereby establishing the absolute configuration of the natural product. In the light of these results, the absolute configuration assigned earlier to (+)-kelsoene needs to be revised as $(+)-\mathbf{1}$.
\end{abstract}

Isolation and structural elucidation of a novel 4-5-5 linearly fused tricyclic sesquiterpene hydrocarbon kelsoene 1, from the tropical marine sponge Cymbastela hooperi collected at Kelso reef, Great Barrier Reef, Australia, was reported in 1997 by Konig and Wright on the basis of incisive spectral analyses. ${ }^{1}$ In 1998, 1 was encountered in an entirely different source, the suspension cultured cells of liverwort Ptychanthus striatus (Lejeuneaceae), seemingly having no phylogenetic relationship with the tropical marine sponge. ${ }^{2}$ The uncommon tricarbocyclic skeleton of $\mathbf{1}$ has been shown $^{2 \mathrm{a}, \mathrm{b}}$ to be biosynthesised from farnesyl pyrophosphate through the intermediacy of germacradienyl and allo aromadendranyl cations. The novel structural features, as well as the interesting biosynthetic origin, aroused our interest in the synthesis of $\mathbf{1}$ and we have recently achieved ${ }^{3}$ its stereoselective total synthesis in racemic form, which fully reaffirmed the assigned
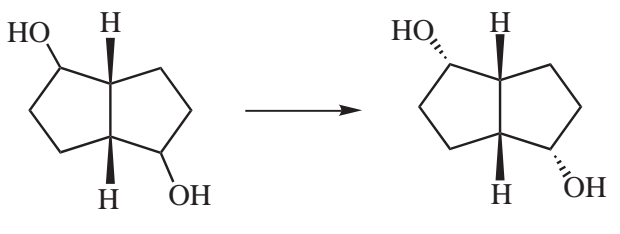

rac-3

stereostructure. Considering that the hydrocarbon kelsoene was not particularly amenable to chiro-optical or NMR probes for configuration determination, we have been concurrently pursuing an enantioselective synthesis of this natural product. However, in a recent communication, Nabeta et al. ${ }^{4 a}$ have reported the determination of the absolute configuration of kelsoene through incisive NMR studies employing a new axially chiral reagent (2'-methoxy-1,1'-binaphthalene-2-carbohydroximoyl chloride, MBCC $)^{4 a, b}$ and formulated it as shown in ent-kelsoene (-)-1. ${ }^{4 \mathrm{~b}}$ In this communication, we describe our efforts towards the syntheses of (+)and (-)-kelsoene 1 from enantiomerically pure $C_{2}$-symmetric diquinane diones (-)-2 and (+)-2, respectively, and show that the absolute configuration assigned earlier ${ }^{4 a}$ to the natural product needs to be reversed and the naturally occurring kelsoene should be correctly represented by $(+)-1$.

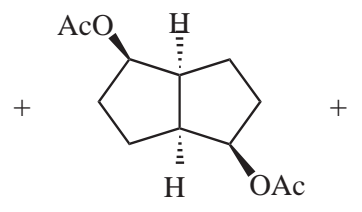

(1S,2R,5S,6R)-4

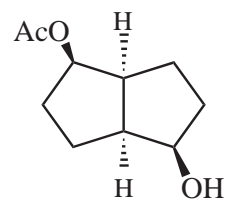

$(1 \mathrm{~S}, 2 \mathrm{R}, 5 \mathrm{~S}, 6 \mathrm{R})-\mathbf{5}$

Scheme 1. Reagents: $( \pm)-3$ (4.0 g, $28.1 \mathrm{mmol})$, vinyl acetate $(19 \mathrm{ml})$, Amano lipase PS-30 (0.8 g), $t$-BuOMe (80 ml), rt, $144 \mathrm{~h}, 85 \%$.

Keywords: enzymes and enzyme reactions; enantiomeric purity; terpenes and terpenoids; configuration.

* Corresponding author. 


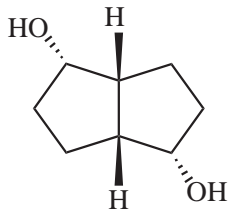

(+)-3

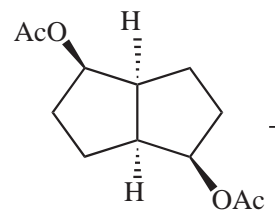

(+)-4
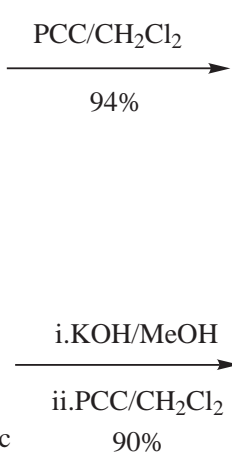

$90 \%$

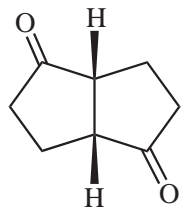

(-)-2

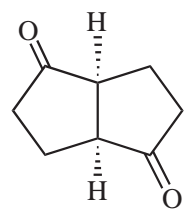

(+)-2
Scheme 2.

The initial objective was to have a convenient access to diquinane diones (-)-2 and (+)-2. endo,endo-cis-Bicyclo[3.3.0]octane-2,6-diol $r a c-3$, readily available from 1,5-cyclooctadiene, on lipase-catalysed enantiomer selective trans-esterification in organic medium, under carefully controlled conditions, led to the isolation of diol (+)-3 ( $>98 \%$ ee, $38 \%$ isolated yield), diacetate $(+)-\mathbf{4}$ (>99\% ee, 33\% isolated yield) and monoacetate $(+)-5$ (72\% ee, 14\% isolated yield) (Scheme 1). ${ }^{5,6}$ Consistent results were obtained when the transesterification of rac-3 (monitored by GLC) was stopped at $40 \%$ conversion to (+)-4, to furnish a chromatographically $\left(\mathrm{SiO}_{2}\right.$ gel) separable mixture (42:40:18) of (+)-3, (+)-4 and $(+)-5$, respectively. ${ }^{5}$ This outcome of enzymatic kinetic resolution was quite satisfying from preparative and enantiomeric purity considerations and readily made available precursors of both the enantiomeric series required for our projected synthesis. While the oxidation of (+)-diol 3 furnished the $C_{2}$-symmetric (-)-dione 2 directly, hydrolysis and oxidation of the (+)-diacetate 4 led to the enantiomeric (+)-dione 2 (Scheme 2). The absolute configuration of chiral dione $\mathbf{2}$ and diol $\mathbf{3}$ has been determined previously. ${ }^{5,6}$

The (-)-dione 2 was elaborated to the natural product kelsoene essentially following the route previously outlined by us $^{3}$ through the intermediacy of the bicyclic $\alpha, \beta$-unsaturated enone (+)-6 and the tricyclic enone (+)-7 (Scheme 3). The key step towards the generation of the tricyclic skeleton of kelsoene was the photochemical [2+2]-photocycloaddition of 1,2-dichloroethylene to (+)-6 from the convex-face and dehalogenation to furnish the cis,anti,cis-fused 5-5-4 ring system (+)-7. Stereoselective transformation of the carbonyl functionality in (+)-7 to an isopropenyl group delivered (+)-kelsoene $1,[\alpha]_{\mathrm{D}}^{25}+67.5\left(c 0.4, \mathrm{CHCl}_{3}\right)$, lit. ${ }^{1,2}[\alpha]_{\mathrm{D}}^{25}+77.1$.
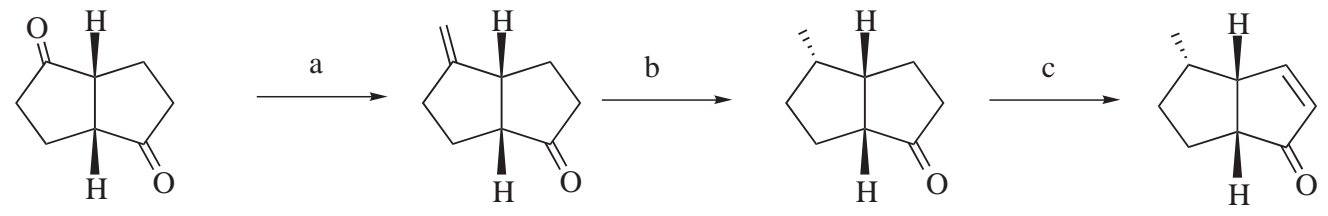

$(-)-2$

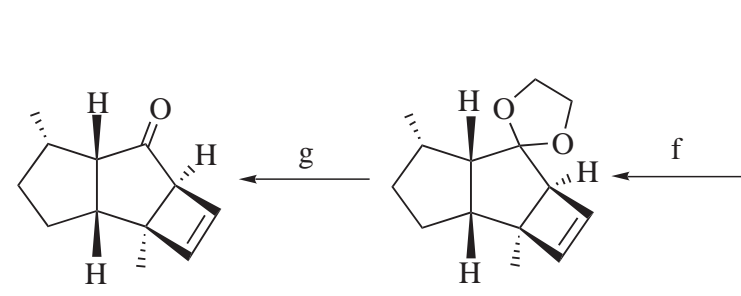

$(+)-7$

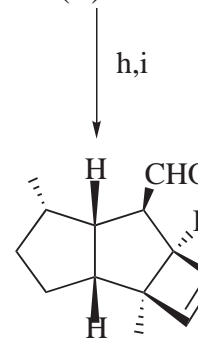
i

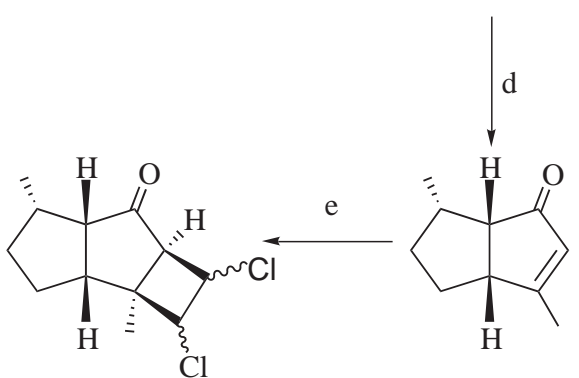

$(+)-6$ 


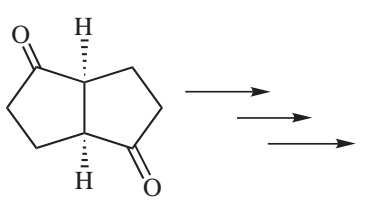

$(+)-2$

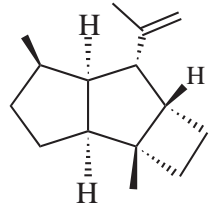

$(-)-1$

\section{Scheme 4.}

The spectral characteristics $\left({ }^{1} \mathrm{H}\right.$ and ${ }^{13} \mathrm{C}$ NMR $)$ of our synthetic sample were exactly identical with the natural product. ${ }^{1}$ Thus, the natural product kelsoene has the absolute configuration depicted in (+)-1.

Initially, not having any inkling of the absolute configuration of the natural product, we also elaborated (Scheme 4) the enantiomerically purer (>99\% ee) dione (+)-2 to ent-kelsoene $1,[\alpha]_{\mathrm{D}}^{25}-76.9$ (c $\left.0.78, \mathrm{CHCl}_{3}\right)$, following the synthetic theme depicted in Scheme 3. This sequence further fortified our deduction of the absolute stereochemistry of the natural product kelsoene.

In short, we have outlined an enantioselective syntheses of the tricyclic sesquiterpene hydrocarbon kelsoene (+)$\mathbf{1}$ and its enantiomer (-)-1, which establish the complete stereostructure of the natural product as (+)-1 and necessitates the revision of the recently assigned ${ }^{4}$ absolute configuration.

\section{Acknowledgements}

The authors would like to thank the SIF facility at the I.I.Sc for the high field NMR spectra and also Professor G. M. Konig, Technical University, Braunschweig, Germany for the comparison spectra of natural kelsoene. K.S. is the recipient of a research fellowship from UGC. The lipase used in this work was a gift from Dr. Y. Hirose of Amano Pharmaceutical Co. Ltd, Japan.

\section{References}

1. Konig, G.; Wright, A. D. J. Org. Chem. 1997, 62, 3837.

2. (a) Nabeta, K.; Yamamoto, K.; Hashimoto, M.; Koshino, H.; Funatsuki, K.; Katoh, K. Chem. Commun. 1998, 1485; (b) Nabeta, K.; Yamamoto, M.; Fukushima, K.; Katoh, K. J. Chem. Soc., Perkin. Trans. 1 2000, 2703.

3. (a) Mehta, G.; Srinivas, K. Tetrahedron Lett. 1999, 40, 4877; (b) Mehta, G.; Srinivas, K. Synlett 1999, 555.

4. (a) Nabeta, K.; Yamamoto, M.; Koshino, H.; Fukui, H.; Fukushi, Y.; Tahara, S. Biosci. Biotechnol. Biochem. 1999, 63, 1772; (b) Fukui, H.; Fukushi, Y.; Tahara, S. Tetrahedron Lett. 1999, 40, 325.

5. Several approaches, ${ }^{6}$ including enzymatic resolution ${ }^{6 \mathrm{a}, \mathrm{b}}$ towards chiral 2,6-disubstituted bicyclo[3.3.0]octanes, have been explored in view of the considerable utility of this ring system in diverse natural product syntheses, e.g. prostanoids. We have also investigated the enzymatic transesterification route to access enantiopure 2,6-disubstituted bicyclo[3.3.0]octanes as described here. However, during the course of these efforts, a similar study appeared in the literature. ${ }^{6 b}$ Procedure for the enzymatic transesterification of rac-diol 3: A mixture of rac-3 (4 g, $28.17 \mathrm{mmol})$, vinyl acetate $(19 \mathrm{ml})$ and Amano lipase PS-30 (0.8 g) in $t$-BuOMe $(80 \mathrm{ml})$ was stirred at room temperature. The reaction was monitored by GLC and stopped at $40 \%$ conversion to the diacetate 4 . The reaction mixture was filtered through a pad of Celite and the filtrate was concentrated. The crude product was charged on a silica gel column and eluted with hexane/ethyl acetate (8:2) to furnish the diacetate $(1 S, 2 R, 5 S, 6 R)-4(2.1 \mathrm{~g}, 33 \%)$ : $[\alpha]_{\mathrm{D}}^{26}+115.0\left(c 2.26, \mathrm{CHCl}_{3}\right)$ for ee $>99 \%$, lit. ${ }^{6 \mathrm{a}, \mathrm{d}}[\alpha]_{\mathrm{D}}^{25}+113.1$ (c 2.26, $\left.\mathrm{CHCl}_{3}\right)$, the monoacetate $(1 S, 2 R, 5 S, 6 R)-5(0.73 \mathrm{~g}$, $14 \%):[\alpha]_{\mathrm{D}}^{27}+44.0\left(c 2, \mathrm{CHCl}_{3}\right)$ for ee $72.2 \%$ (obtained by conversion to the (-)-diol 3) and the diol $(1 R, 2 S, 5 R, 6 S)-3$ $(1.52 \mathrm{~g}, 38 \%):[\alpha]_{\mathrm{D}}^{27}+49.5\left(c 2, \mathrm{CHCl}_{3}\right)$ for ee $\left.>99 \%\right)$, lit. ${ }^{6 \mathrm{~b}}$ $[\alpha]_{\mathrm{D}}^{20}+42.5\left(c 1, \mathrm{CHCl}_{3}\right)$ for ee $98.5 \%$.

6. (a) Djadchenko, M. A.; Pivnitsky, K. K.; Theil, F.; Schick, H. J. Chem. Soc., Perkin Trans. 1 1989, 2001; (b) Lemke, K.; Ballschuh, S.; Kunath, A.; Theil, F. Tetrahedron: Asymmetry 1997, 8, 2051; (c) Perard-Viret, J.; Rassat, A. Tetrahedron: Asymmetry 1994, 5, 1; (d) Djadchenko, M. A.; Melnikova, V. I.; Pivnitsky, K. K. Zh. Obshch. Khim. 1986, 56, 2143 . 